\title{
Synergistic effect of the TLR5 agonist CBLB502 and its downstream effector IL-22 against liver injury
}

\author{
Nicolas Melin 1,2, Daniel Sánchez-Taltavull, ${ }^{1,2}$, René Fahrner ${ }^{1,2,4}$, Adrian Keogh ${ }^{1,2}$, Michel Dosch (1,2, Isabel Büchi ${ }^{1,2}$, \\ Yitzhak Zimmer ${ }^{1,3}$, Michaela Medová (i] ${ }^{1,3}$, Guido Beldi $\mathbb{B}^{1,2}$, Daniel M. Aebersold ${ }^{1,3}$, Daniel Candinas ${ }^{1,2}$ and \\ Deborah Stroka (iD ${ }^{1,2}$
}

\begin{abstract}
The toll-like receptor 5 (TLR5) agonist, CBLB502/Entolimod, is a peptide derived from bacterial flagellin and has been shown to protect against radiation-induced tissue damage in animal models. Here we investigated the protective mechanism of CBLB502 in the liver using models of ischemia-reperfusion injury and concanavalin A (ConA) induced immuno-hepatitis. We report that pretreatment of mice with CBLB502 provoked a concomitant activation of NF-KB and STAT3 signaling in the liver and reduced hepatic damage in both models. To understand the underlying mechanism, we screened for cytokines in the serum of CBLB502 treated animals and detected high levels of IL-22. There was no transcriptional upregulation of $\mathrm{IL}-22$ in the liver, rather it was found in extrahepatic tissues, mainly the colon, mesenteric lymph nodes (MLN), and spleen. RNA-seq analysis on isolated hepatocytes demonstrated that the concomitant activation of NF-KB signaling by CBLB502 and STAT3 signaling by IL-22 produced a synergistic cytoprotective transcriptional signature. In IL-22 knockout mice, the loss of IL-22 resulted in a decrease of hepatic STAT3 activation, a reduction in the cytoprotective signature, and a loss of hepatoprotection following ischemiareperfusion-induced liver injury. Taken together, these findings suggest that CBLB502 protects the liver by increasing hepatocyte resistance to acute liver injury through the cooperation of TLR5-NF-KB and IL-22-STAT3 signaling pathways.
\end{abstract}

\section{Introduction}

Hepatic diseases are a major health care burden worldwide. In many cases, the necessary treatment option for end-stage hepatic disease is liver transplantation, however, less than $10 \%$ of the global transplantation needs are currently met ${ }^{1}$. New methods of providing protection against hepatic injury, thus ameliorating continued liver dysfunction and failure, may provide support for this shortcoming.

The innate immune system recognizes molecules shared by pathogens through germ-line encoded toll-like receptors (TLR). When activated, TLRs recruit toll/

\footnotetext{
Correspondence: Deborah Stroka (deborah.stroka@dbmr.unibe.ch)

${ }^{1}$ Department for BioMedical Research, Inselspital, Bern University Hospital,

University of Bern, 3008 Bern, Switzerland

${ }^{2}$ Visceral Surgery and Medicine, Inselspital, Bern University Hospital, University

of Bern, 3008 Bern, Switzerland

Full list of author information is available at the end of the article

Edited by S. He
}

interleukin-1 receptor (TIR) domain-containing adapter proteins such as myeloid differentiation primary response 88 (MyD88) and TIR-domain-containing adapterinducing interferon- $\beta$ (TRIF), which initiate signal transduction pathways that culminate in the activation of nuclear factor kappa B (NF-kB), interferon regulatory factors (IRF), or mitogen-activated protein kinases $(\text { MAPK })^{2}$. Regulation of TLR signaling is important as its sustained activation can exacerbate an inflammatory response resulting in cellular and tissue damage ${ }^{2}$, such as the amplification of inflammation in rheumatoid arthritis $^{3}$, inflammatory bowel disease ${ }^{4}$, acute injury provoked by ischemia-reperfusion ${ }^{5}$, and tissue fibrosis ${ }^{6}$. On the other hand, judicious activation of TLRs can be beneficial for tissue repair and protection by activating cytoprotective, anti-inflammatory signaling pathways ${ }^{6,7}$.

TLR5 is activated via the engagement of leucine-rich repeat (LRR) ectodomains of TLR5 with its natural ligand,

\section{(c) The Author(s) 2021}

(c) (i) Open Access This article is licensed under a Creative Commons Attribution 4.0 International License, which permits use, sharing, adaptation, distribution and reproduction cc) in any medium or format, as long as you give appropriate credit to the original author(s) and the source, provide a link to the Creative Commons license, and indicate if changes were made. The images or other third party material in this article are included in the article's Creative Commons license, unless indicated otherwise in a credit line to the material. If material is not included in the article's Creative Commons license and your intended use is not permitted by statutory regulation or exceeds the permitted use, you will need to obtain permission directly from the copyright holder. To view a copy of this license, visit http://creativecommons.org/licenses/by/4.0/. 
the three helices of the flagellin D1 domain ${ }^{8}$. TLR5 agonists were shown to limit ischemia-reperfusion injury (IRI) in the kidney ${ }^{9}$ and to decrease graft versus host disease $^{10}$. The beneficial effect of TLR5 activation has been intensely investigated in the context of radiationinduced toxicity, resulting in the development of the optimized flagellin derivative, Entolimod, or CBLB502 $(\mathrm{CBLB})^{11}$. Its proposed mechanism of protection is the increased survival of intestinal and hematopoietic stem cells by reducing inflammation and promoting antiapoptotic and redox modulating pathways ${ }^{12}$. Based on its immunostimulatory effect and protective properties, the use of CBLB has advanced towards clinical trials to promote antitumor responses, to reduce radiotherapy and/or chemotherapy-induced side effects, and to be used as a potentiating adjuvant during vaccinations (www. clinicaltrial.gov. trial ref: NCT02715882, NCT03063736, NCT01527136, NCT01728480).

Based on the studies demonstrating its protective activity in normal tissue, we investigated the mechanism of CBLB's protection in the liver. We used two hepatic injury models; IRI as a ROS and immune-induced hepatic damage model ${ }^{13}$, and autoimmune-hepatitis induced by concanavalin A (ConA) as a hepatocyte-apoptosis model. Our results demonstrate that CBLB protects against hepatic damage by protecting against parenchymal cell death. The hepatoprotective effect is not solely achieved by TLR 5 activation on hepatocytes but requires concomitant signal transducer and activator of transcription 3 (STAT3) signaling induced by remote CBLB-mediated IL22 secretion.

\section{Materials and Methods}

\section{Animal models}

Animals were housed in specific pathogen-free conditions in accord with the Swiss veterinary office. Eight to twelve weeks old C57BL/6J male and female mice (Harlan Laboratories B.V., Horst, Netherlands), MyD88/TRIF double knockout mice ${ }^{14}$, and IL-22 knockout mice ${ }^{15}$ were acclimatized in a temperature-controlled room with a 12 hours dark/light cycle and ad libitum access to water and chow. CBLB was dissolved in saline and injected i.p. $(5 \mu \mathrm{l} / \mathrm{g}$ mouse) to reach a final concentration of $0.2 \mathrm{mg} / \mathrm{kg}$ CBLB or an equivalent volume of saline (vehicle) in a randomized (equal distribution of animals amongst cages) and blinded (CBLB vs. Saline) $2 \mathrm{~h}$ prior to the IRI or ConA challenge. IRI was induced by depriving the left lobe of blood inflow for $1 \mathrm{~h}$ according to the previously described procedure $^{16}$. Serum was collected $6 \mathrm{~h}$ post-reperfusion and mice were sacrificed at $24 \mathrm{~h}$. Tissue from the left lobe was fixed in $4 \%$ PFA for $48 \mathrm{~h}$ and snap-frozen. ConA injury was provoked following a published protocol ${ }^{17}$ with $12 \mathrm{mg} / \mathrm{kg}$ of ConA injected i.v. (Sigma-Aldrich, Darmstadt, Germany, \#C7642). Mice were sacrificed after $8 \mathrm{~h}$, serum was collected, the left liver lobe was fixed in $4 \%$ PFA for $24 \mathrm{~h}$, and the right lobe was snap frozen. Animal procedures were approved by the Veterinary Office of the Canton Bern in line with institutional and standard protocols for the care and use of laboratory animals.

\section{Measurement of serum alanine transaminase}

Post-injury serum samples were collected to assess the extent of liver injury using alanine transaminase assay (P800; Modular Analytics EVO, Roche, Mannheim, Germany). The results were analyzed and displayed with GraphPad Prism software, representing the mean and standard deviation and analyzed using two-sided Student's $t$-test. (GraphPad Software Inc., La Jolla, CA, USA).

\section{Histology}

Hematoxylin and eosin staining and Gr1 immunohistochemistry (eBioscience, San Diego, CA, USA, 4-593185) were done following standard protocols previously described ${ }^{16}$ and imaged with the Panoramic ${ }^{\circledR} 250$ Flash III slide scanner (3DHISTECH, Budapest, Hungary) using a 20x objective and the \#DHISTECH acquisition software at room temperature.

\section{Myeloperoxidase assay}

Proteins were extracted from total liver tissue and myeloperoxidase (MPO) activity assay was carried out as previously described ${ }^{16}$. The results were analyzed and displayed with GraphPad Prism software, representing the mean and standard deviation and analyzed using twosided Student's $t$-test. (GraphPad Software Inc., La Jolla, CA, USA).

\section{Flow cytometry}

Liver leukocytes were isolated by mechanical dissociation as described previously ${ }^{18}$. The following fluorescently-labeled antibodies targeting mouse antigens were used: CD45-BUV (BD Biosciences, Franklin Lakes, NJ, USA, 30-F11), CD3-FITC (BioLegend, San Diego, CA, USA, 17A2), NK1.1-APC-eFluor780 (eBioscience, PK136), CD25-APC (ebioscience, PC61.5), and CD69PeCy7 (BD Biosciences, H1.2F3). Cells were acquired on a SORP LSRII (BD Pharmingen Inc., San Diego, CA) and flow cytometry data were analyzed using FlowJo software (FlowJo, LLC) following the gating strategy given in Supplementary Fig. 1. The results were analyzed and displayed with GraphPad Prism software, representing the mean and standard deviation and analyzed using twosided Student's $t$-test. (GraphPad Software Inc., La Jolla, CA, USA).

\section{Serum cytokine measurements}

Circulating cytokines and chemokines were assessed using the magnetic bead array based ProcartaPlex mouse 
basic kit (\#EXP010-20440-901) in combination with the targets kit (Supplementary Table 1), following the manufacturer's instructions (eBioscience) and measured using Luminex instrument (xMAP Technology). The results were analyzed and displayed with GraphPad Prism software, representing the mean and standard deviation. (GraphPad Software Inc., La Jolla, CA, USA).

\section{RNA isolation and quantification}

RNA was isolated from snap-frozen tissue using TRIzol (Sigma), quantified, and cDNA was generated using Omniscript RT Kit 200 (Qiagen, Hilden, Germany) as previously described ${ }^{19}$. qPCRs were performed using TaqMan gene expression assays for Il22, Tbp, Socs3, Hmox1, and Tnfaip3 (Thermo Fisher Scientific; Supplementary Table 2). Il22 RNA was quantified following the absolute quantification methods described by Pfaffl et al. ${ }^{20}$. Tbp PCR product was cloned in a pGEM ${ }^{\circledR}-\mathrm{T}$ Easy Vector (Promega, Madison, WI, USA, \#A3600), the plasmid was amplified using the One Shot TOP10 Chemically Competent E. coli (Thermo Fisher Scientific) and purified using the PureYield MaxiPrep (Promega). Il22 was quantified based on the Tbp standard, assuming a perfect PCR efficacy. For the other targets, relative changes in mRNA were calculated with the $\Delta \Delta \mathrm{Ct}$ method using Tbp as a control gene. The results were analyzed and displayed with GraphPad Prism software, representing the mean and standard deviation and analyzed using two-sided Student's $t$-test. (GraphPad Software Inc., La Jolla, CA, USA).

\section{Western blot}

Western blots were done following previously used protocols $^{21,22}$ and the primary antibody for I $\mathrm{KB} \alpha$ (Santa Cruz Biotechnology, Dallas, TX, USA \#sc371), and STAT3 (\#9139), phospho-STAT3-Tyr705 (\#9145) or TBP (\#8415) all from Cell Signaling Technology (Danvers, MA, USA), were used at a 1:1000 dilution, and the secondary antibodies were anti-rabbit-HRP (Dako, Agilent technology, Santa Clara, CA, USA) or anti-rabbit-680LT or antimouse-800CW (LI-COR Bioscience, Lincoln, NE, USA). Band densities were analyzed using ImageJ and displayed with GraphPad Prism software, representing the mean and standard deviation and analyzed using two-sided Student's $t$-test. (GraphPad Software Inc., La Jolla, CA, USA).

\section{Hepatocyte isolation and culture}

C57BL/6 mouse hepatocytes were isolated in situ with collagenase perfusion as previously described ${ }^{23}$, and cultured on rat tail collagen coated plate in arginine free Williams E media supplemented with $40 \mathrm{mM}$ ornithine, $100 \mathrm{iU} / \mathrm{ml}$ insulin, $2 \mathrm{mg} / \mathrm{ml}$ hydrocortisone, penicillin, and streptomycin. Twenty-four hours after plating, the hepatocytes were treated with CBLB $(4 \mathrm{ng} / \mathrm{ml}$ Cleveland BioLabs, Buffalo, NY, USA) and/or IL-22 (1 ng/ml; PeproTech, Rocky Hill, NJ, USA, \#210-22) in combination.

\section{Transcriptomic and pathway analysis}

RNA was extracted from snap-frozen tissue with RNeasy Micro Kit (Qiagen; 74004), quantified by Qubit BR assay (Thermo Fisher Scientific) and quality assessed by Fragment Analyzer (Advanced Analytical, Parkersburg, WV, USA) using Standard Sensitivity RNA Analysis Kit (Advanced Analytical, \#DNF-471). The library was prepared without size exclusion with the TruSeq Stranded mRNA kit (Illumina, San Diego, CA, USA, \#20020594). Qubit dsDNA HS (Thermo Fisher Scientific; \#Q32851) and Fragment Analyzer NGS Kit (Advanced Analytical; \#DNF-474) were used for library quality control. Finally, the samples were sequenced on a NovaSeq6000 (Illumina), paired-end $1 \times 50 \mathrm{bp}$ on one lane. Fastq files were aligned to the mouse reference genome $\mathrm{mm} 10$ with HISAT2 ${ }^{24}$. The resulting SAM files were transformed into sorted BAM files with SAMTOOLS ${ }^{25}$. Further analysis was done using the RStudio software

The counts were counted from the bam files with featureCounts $R$ function of the $R$ package Rsubread ${ }^{26}$. Differentially expressed genes (DEG) were assessed using DESeq $2^{27}$. In every condition (control, CBLB, IL-22, CBLB + IL-22), a paired comparison within each isolation replicate was performed. In order to visualize the data in two dimensions, we transformed the data into reads per million (RPM) and performed a principal component analysis with the $\mathrm{R}$ function prcomp. The new resulting coordinates (PC1 and $\mathrm{PC} 2$ ) were translated to move the Control to the position 0,0 , that is $\mathrm{f}_{\mathrm{i}}(\mathrm{x}, \mathrm{y})=\left(\mathrm{x}_{\mathrm{i}}-\mathrm{c}_{\mathrm{x}, \mathrm{i}}, \mathrm{y}_{\mathrm{n}}-\right.$ $\left.c_{y, i}\right)$, with $(i=1,2,3)$ denoting the hepatocytes isolation experiment. Gene Log10( $p$ value) and Log2(fold change) $(\log 2 \mathrm{FC})$ changes for each condition were visualized using volcano plots with the gene with an adjusted $p$ value $<$ 0.05 and $-1<\log 2 \mathrm{FC}>1$ were colored in green. A heatmap figure was produced using normalized gene expression with the maximum expression set to 1 and the minimum to 0 , using the function heatmap. 2 from the $\mathrm{R}$ package $^{28}$, with complete linkage using correlation distance.

The stackplots showing the pathways were performed with a custom $R$ script. First, we selected the ten most significant families of pathways identified by Metascape. Second, we obtained all the genes involved with the $R$ function gconvert of the $\mathrm{R}$ package gprofiler2. Third, we computed the intersection between the gene lists and the statistically significant differentially expressed and colored according to $\log 2 \mathrm{FC}$. The significance of each pathway was assessed by Metascape. For the p53 signaling pathway (mmu04115), the genes were manually included from the 
Metascape output. The bar plots showing the 20 most significant families of pathways were done with Metascape.

\section{Results}

TLR5 agonist CBLB is hepatoprotective in vivo

The effect of CBLB against oxidative stress generated by ischemia-reperfusion and by immune-driven hepatic damage caused by ConA was tested in mice pretreated with CBLB for $2 \mathrm{~h}$ (Fig. 1A, F). Following $1 \mathrm{~h}$ of partial liver ischemia and $6 \mathrm{~h}$ reperfusion, serum ALAT levels elevated to 2883I.U. in control animals, whereas ALAT levels only increased to 688I.U. in animals pretreated with CBLB (Fig. 1B). Twenty-four hours after reperfusion, the livers of CBLB treated mice displayed smaller necrotic areas (Fig. 1C), which was accompanied by a reduction of MPO activity (Fig. 1D) resulting from the decreased neutrophil infiltration (Fig. 1E). In the ConA model, CBLB pretreated animals had a tenfold decrease of ALAT serum levels (from 11186I.U. to 1068I.U.) following ConA challenge compared to control (Fig. 1G), and a decrease in necrotic areas compared to controls (Fig. $1 \mathrm{H})$. T cells and NKT cells are the primary effector cells in mediating ConA injury ${ }^{17,29}$. In control mice treated with ConA, there was an increase in the percentage of $\mathrm{T}$ cells in the infiltrating CD45+ cells (Fig. 1I), and a reduced percentage of NKT cells (Fig. 1J). In both T and NKT cells, there was an increase in their activation markers CD25 and CD69, with more double-positive cells present (Fig. 1K, L). CBLB did not alter the percentage of infiltrating immune cells nor did it modify their activation marker expression upon ConA challenge (Fig. 1I-L). Taken together, our in vivo data suggests that pretreatment with CBLB prevents hepatic damage in both IRI and ConA liver injury, as determined by the early reduction in serum ALAT levels. As there was no difference in the NKT and $\mathrm{T}$ cell infiltration and activation in the ConA model, this suggested that CBLB did not alter the immune cell infiltration and activation, but rather may enhance the resistance of hepatocytes to harmful stimuli.

\section{CBLB induces hepatic STAT3 activation and remote IL-22 production}

Previous reports have shown that CBLB activates both NF- $\kappa B$ and STAT3 signaling pathways in vivo ${ }^{12}$. As CBLB is known to directly activate the NF- $\kappa B$ pathway via TLR5 signaling ${ }^{30}$, we explored the origin of the CBLBinduced STAT3 activation in the liver. Two hours postinjection, CBLB induced the phosphorylation of tyrosine 705 of STAT3 in liver tissue (Fig. 2A) As STAT3 signaling is mainly mediated by Janus kinases upon cytokine stimulation $^{31}$, we evaluated the serum of mice for 22 cytokines and chemokines 2 and $6 \mathrm{~h}$ following CBLB exposure. TLR5 activation did not increase the serum level of 13 of the tested cytokines, IL-1 $\beta$, IL-4, IL-10, IL12p70, IL-13, IL-15/IL-15R, IL-17A, IL-17F, IL-23, IL-33, $\mathrm{TNF} \alpha, \mathrm{INF} \alpha$, and INF $\gamma$ (Supplementary Fig. 2), whereas nine other cytokines were elevated in the serum with a peak $2 \mathrm{~h}$ post-injection. There was a minor increase of the chemokine CCL5 and a greater than tenfold increase of CCL2, CXCL1, and CXCL2 to $\sim 100 \mathrm{pg} / \mathrm{ml}$. The proinflammatory cytokine IL- $1 \alpha$ was increased to $50 \mathrm{pg} / \mathrm{ml}$ and IL- 6 and IL-18 were increased to around $200 \mathrm{pg} / \mathrm{ml}$. For cytokines involved in wound healing, IL-5 showed a minor response, whereas serum levels of IL-22 reached an average level of $1670 \mathrm{pg} / \mathrm{ml}$ (Fig. 2B). IL-22 is an antifibrotic cytokine, that prevents liver injury, promotes liver regeneration $^{32,33}$, and was shown to protect against both hepatic IRI and ConA-induced liver damage ${ }^{34,35}$. Consequently, we considered IL-22 as a possible contributor to the hepatoprotective effect of TLR5 activation.

To assess if the increase of IL-22 in the serum of CBLB treated mice was due to TLR5 signaling, CBLB was administered to MyD88/TRIF double KO mice. There was no increase in serum IL-22 levels, suggesting that CBLB increased levels of IL-22 in a TLR5-MyD88-TRIFdependent manner (Fig. 2C). We next questioned if the increase of IL-22 serum levels was a direct and localized response from the liver. We observed no transcriptional increase of IL-22 in the liver tissue of mice 2 and $6 \mathrm{~h}$ following CBLB injection (Fig. 2D). To then determine the source of IL-22 detected in serum, we screened 17 organs for IL-22 mRNA expression following CBLB exposure. There were no detectable levels of IL-22 mRNA in all organs prior to CBLB injection, nor in the pancreas, bone marrow, skin, kidney, brain, muscle, heart, and blood $2 \mathrm{~h}$ post-injection (Supplementary Fig. 3). In the colon and MLN, there was a strong increase in IL-22 mRNA, and to a smaller extent also in the intestine, spleen, thymus, lung, salivary gland, and stomach. After $6 \mathrm{~h}$, IL-22 mRNA levels were lower in all organs, with the exception of the MLN that showed a 2.5 times higher level of IL-22 transcript compared to values at $2 \mathrm{~h}$ (Fig. 2D and Supplementary Fig. 3). Taken together, our data demonstrate that in addition to NF- $\mathrm{KB}$ activation, STAT3 is activated in the liver of mice exposed to CBLB. STAT3 activation can be a result of TLR5-induced cytokine expression, particularly IL-22. We furthermore conclude that IL-22 is not produced in the liver, but rather remotely in the colon and MLN.

\section{Pathway analysis of hepatocytes stimulated with CBLB and IL-22}

We next questioned if CBLB and/or IL-22 directly alter the transcriptional profile of hepatocytes thus explaining its hepatoprotective effect. We first validated that hepatocytes have a direct response to TLR5 activation and IL22 by treating primary mouse hepatocytes with CBLB and IL-22 and measuring downstream receptor signaling. 


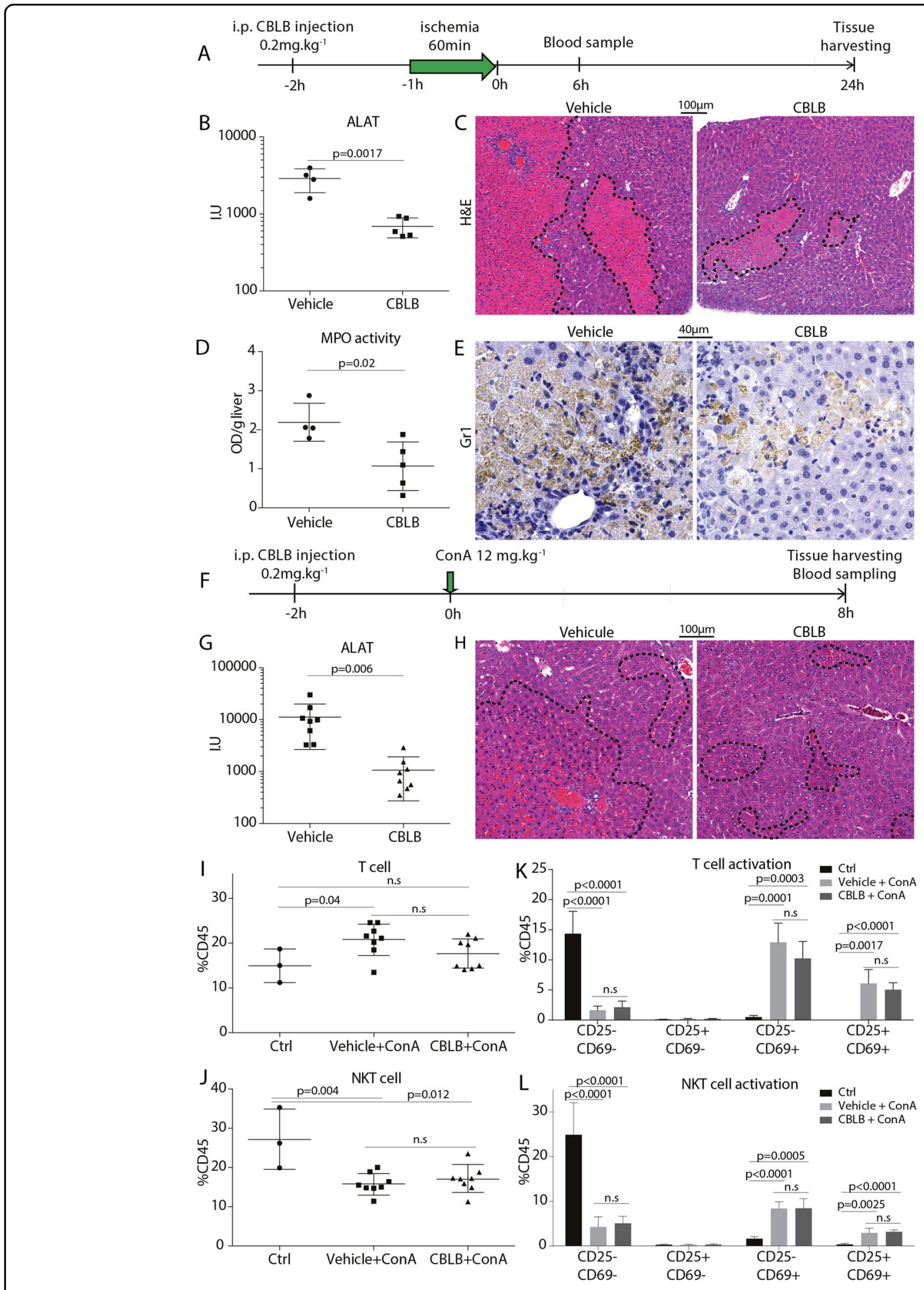

Fig. 1 CBLB decreases hepatic damage. A Experimental plan of ischemia-reperfusion study, vehicle $n=4$, CBLB $n=5$ mice. B Serum ALAT $6 \mathrm{~h}$ after reperfusion. C Representative H\&E image of liver tissue $24 \mathrm{~h}$ post-reperfusion. D Tissue myeloperoxidase activity $24 \mathrm{~h}$ post-reperfusion. E

Representative images of liver Gr1 immunohistochemistry $24 \mathrm{~h}$ post-ischemia-reperfusion. $\mathbf{F}$ Experimental plan of ConA model, vehicule $n=8$, CBLB $n=8$. G Serum ALAT $8 \mathrm{~h}$ after ConA challenge. $\mathbf{H}$ Representative H\&E image of liver tissue $8 \mathrm{~h}$ following ConA injection. FACS analysis of I T cell and J NKT cells frequency represented as a percentage of CD45 + cells extracted from the liver $8 \mathrm{~h}$ ConA injection. FACS analysis of the activation frequency of $\mathbf{K}$ T cell and $\mathbf{L}$ NKT cell markers CD25 and CD69. Frequencies are represented as a percentage of CD45 + cells extracted from liver $8 \mathrm{~h}$ post-ConA injection. 

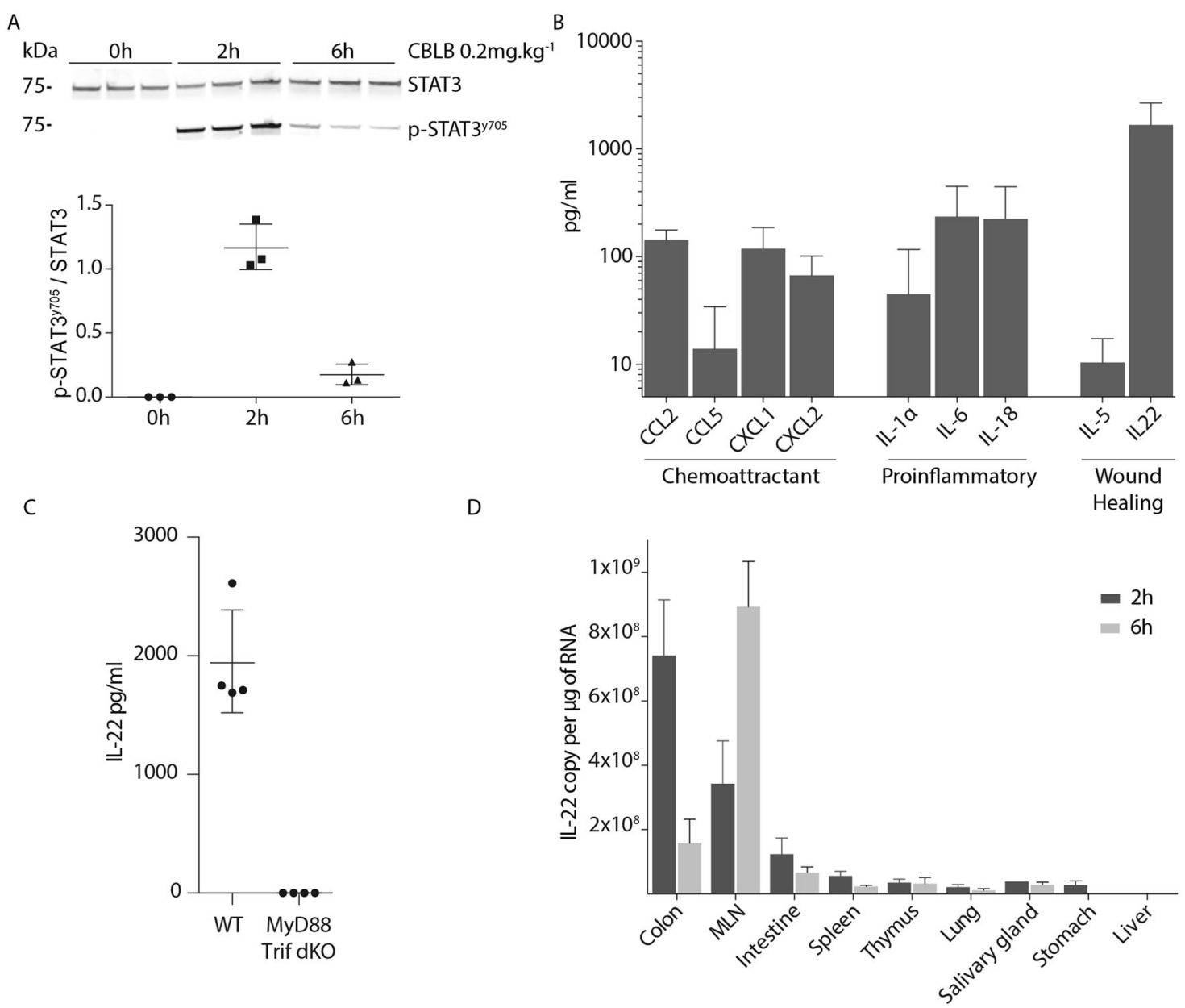

Fig. 2 CBLB induces a remote IL-22 release that triggers hepatic STAT3 signaling. A Western blot analysis of liver STAT3 and STAT3 phosphorylated at tyrosine 705 at control, $2 \mathrm{~h}$, and $6 \mathrm{~h}$ following CBLB injection, and its STAT3 phosphorylated at tyrosine 705/STAT3 ratio, $n=3$ mice per group. B Analysis of mouse serum $2 \mathrm{~h}$ post-CBLB injection, $n=7$ mice. C IL-22 serum levels in WT and MyD88/Trif double knockout mice $2 \mathrm{~h}$ postCBLB injection, $n=4$ mice per group. D RNA expression in various tissue 2 and $6 \mathrm{~h}$ post-CBLB injection, $n=3$ mice per group.

CBLB led to the activation of the NF- $k B$, indicated by the degradation of its inhibitory protein IKB $\alpha$ but did not lead to STAT3 phosphorylation (Fig. 3A and Supplementary Fig. 4). IL-22 induced the phosphorylation of STAT3 on tyrosine 705, signifying activation of STAT3 transcriptional activity but did not result in the activation of NF- $\mathrm{KB}$ (Fig. 3A and Supplementary Fig. 4). The downstream transcriptional response was measured by bulk RNA-seq in hepatocytes isolated from three mice that were treated with either CBLB or IL-22 alone or in combination (CBLB + IL-22) for $2 \mathrm{~h}$. The first principal component (PC1) indicated a strong combinatorial effect of CBLB + IL-22, which clustered to the right compared to the control, IL-22 and CBLB treated cells, clustered to the left. In the second principal component (PC2), differences between control and CBLB groups could be observed (Fig. 3B). We next assessed the distribution of statistically significant DEG in the three treatment conditions compared to their untreated controls. CBLB treated hepatocytes displayed 1771 downregulated and 2045 upregulated DEG. The effect of IL-22 on hepatocytes was minor; only 52 downregulated and 100 upregulated DEG were observed. The combination of CBLB + IL-22 resulted in 1184 downregulated and 1587 upregulated DEG. Although the exposure to IL-22 only induced a small effect, adding IL-22 to CBLB altered the response to CBLB alone. Indeed, the addition of IL-22 to CBLB decreased the overall number of DEG and considerably increased their fold change intensity (Fig. 3C).

Next, we questioned if the changes in the DEG between the groups were the same. Gene overlap analysis was performed using the multiple gene list function on Metascape and was then displayed in a Circos plot. IL-22 had no unique DEG; all were shared with the CBLB + IL22 group. While CBLB and CBLB + IL-22 had shared DEG, both also had a majority of non-overlapping, unique 
A

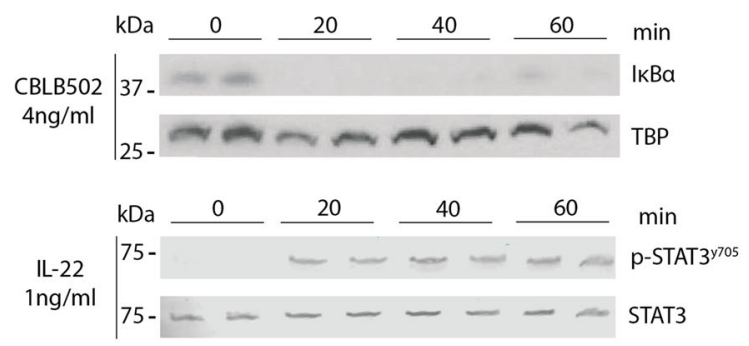

C

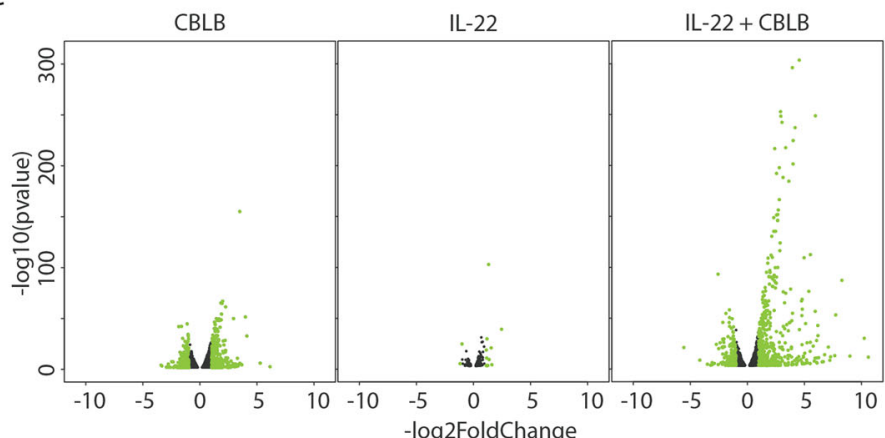

B

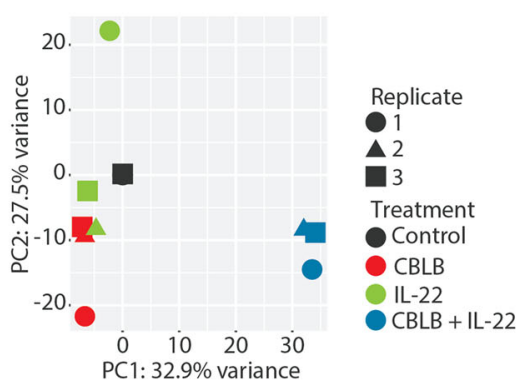

D

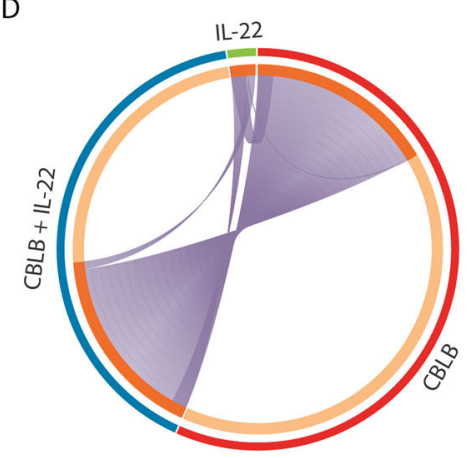

D

$\mathrm{F}$

E

Pathway

- $\log 10$ (pvalue)

$\begin{array}{lllll}0 & 10 & 20 & 30 & 40\end{array}$

monocarboxylic acid metabolic process. 45

small molecule catabolic process. $23 \quad 102$

cellular amino acid metabolic process. 33

apoptotic signaling pathway. 120 p53 signaling pathway.

cellular ketone metabolic process.

drug metabolic process. 40

positive regulation of cell death. 117

negative regulation of cell cycle. 82

response to external stimulus 123

inflammatory response.

response to hormone.

blood vessel development

epithelial cell apoptotic process.

positive regulation of hemopoiesis.

regulation of cytokine production.

T-helper 1 type immune response

leukocyte activation / immune response.

Cytokine-mediated signaling pathway.

monocarboxylic acid metabolic process.

inflammatory response 130

cytokine production 146

Cytokine-mediated signaling pathway. 94

positive regulation of cell migration. 107

TNF signaling pathway.

response to molecule of bacterial origin. 88

regulation of cell adhesion 120

positive regulation of cell death. 124

apoptotic signaling pathway. 112

I-kappaB kinase / NF-kappaB signaling.

\begin{tabular}{|r|r|}
\hline 123 & 83 \\
\hline 10 & 5 \\
\hline 17 & 16 \\
\hline 9 & 4 \\
\hline 11 & 1 \\
\hline 18 & 3 \\
\hline & 2 \\
\hline
\end{tabular}

Color key \& histogram

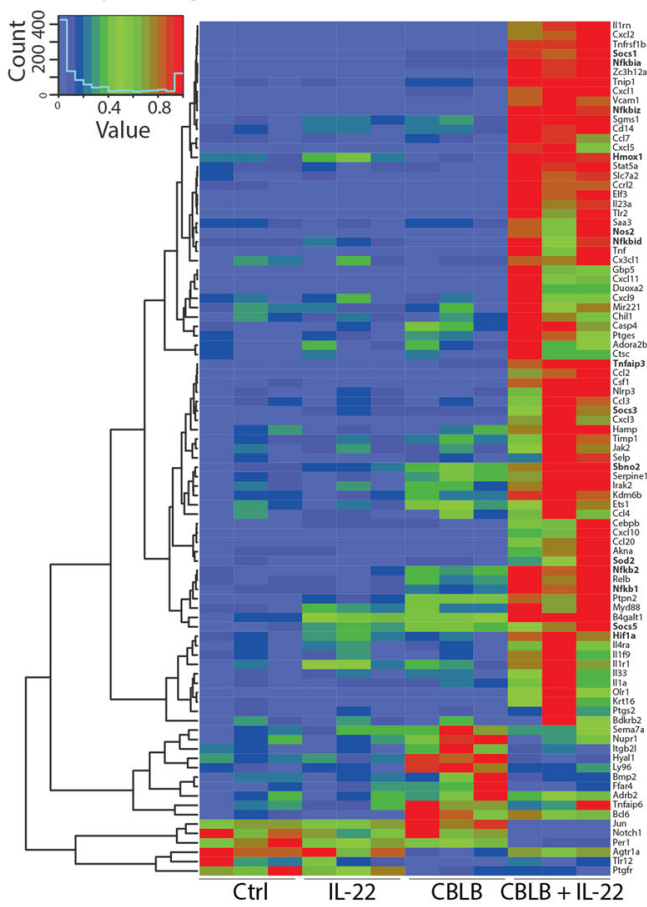

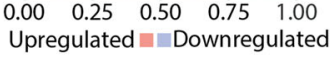

Fig. 3 CBLB and II-22 induce hepatocyte NF-KB and STAT3 signaling, respectively. A Western blot analysis of hepatocytes for IKBa normalized by TBP and STAT3 phosphorylated at tyrosine 705 normalized by STAT3 at control, 20, 40, and 60 min after CBLB or IL-22 exposure, $n=2$ replicates per time point. B The PCA of the transcriptomic signature of hepatocytes treated for $2 \mathrm{~h}$ with either CBLB in red, IL-22 in green, CBLB + IL-22 in blue, or control in black. The isolation replicates are indicated with the same shape, $n=3$ replicates. $\mathbf{C}$ Volcano plot displaying the differentially expressed genes (DEG) of each condition compared to control, showing the DEG with a $-1<\log 2 \mathrm{FC}>1$ in green. $\mathbf{D}$ Circos plot showing on the outer ring the treatment groups and on the inner ring the DEG. DEG that are shared by the different groups are indicated in dark orange, DEG unique to each group are in light orange. Each gene shared between the different groups is indicated by purple lines. E Bar graph presenting the ten most regulated pathways of each condition, with the number of DEG upregulated in red or downregulated in blue for each condition. $\mathbf{F}$ Heat map of the inflammatory response pathway showing the DEG with $a-1<\log 2 F C>1$, with genes known to be hepatoprotective in IRI or ConA models in bold. 
DEG (Fig. 3D) which is further displayed as a heatmap in Supplementary Fig. 5.

To evaluate the functional consequences elicited by each treatment, we performed a pathway enrichment analysis using Metascape which generated a list of 40 pathways associated with up- and down-regulated DEG for each condition (Supplementary Fig. 6). Based on $p$ values ranging from $-\log (p) 8.4$ to 19.6 , the ten most regulated pathways in hepatocytes treated with CBLB alone were comprised of downregulated genes involved in metabolic processes, and upregulated genes are involved in apoptotic signaling and cell cycle regulation. Interestingly, aside from the upregulation of genes involved in the TNF signaling pathway, we did not identify the regulation of genes involved in inflammatory, cytokine, or immune response pathways (Fig. 3E and Supplementary Fig. 6). Pathway enrichment analysis of DEG in IL-22 treated hepatocytes resulted in pathways with $-\log (p)$ values below 10. The ten most regulated pathways showed an upregulation of genes associated with inflammation, cytokine and immune response, and a downregulation of genes associated with metabolism (Fig. 3E and Supplementary Fig. 6).

The most significantly regulated pathways, with $p$ values ranging from $-\log (p) 15.5$ to 36.2 were found in the CBLB + IL-22 group and were associated with the upregulation of genes involved in NF- $\mathrm{KB}$ and apoptotic signaling, as well as inflammatory, cytokine and immune response pathways. The $-\log (p)$ values of the pathways associated with downregulated genes in the CBLB + IL-22 group were all below 9.5. (Fig. 3E and Supplementary Fig. $6)$.

We further investigated the pathway with the highest $p$ value across all groups, which was the inflammatory response, and computed a heat map for all the DEG with a fold change below 0.5 or above 2 . Strikingly, the combination of CBLB + IL-22 resulted in the upregulation of a large gene set (Fig. 3F). Among those genes strongly upregulated by CBLB + IL-22, we found inhibitors of inflammatory processes such as Nfkbia, Nfkbid, Nfkbiz, Sbno2, Socs1, Socs3, Socs5, and Tnfaip3, and genes regulating redox homeostasis like Hif1a, Hmox1, Nos2, and Sod2, and antiapoptotic genes Tnfaip3 (Supplementary Figs. 7 and 8). The transcriptomic analysis suggests that the combination of CBLB + IL-22 incites a cytoprotective signature that is not accomplished by either of the two treatments alone.

\section{IL-22 is required for CBLB-induced hepatoprotection}

From the data presented above, IL-22 appeared to have a key role in CBLB-mediated hepatoprotection. For further validation, we assessed the hepatoprotective effect of CBLB in IL-22 knockout (IL-22 ${ }^{-1-}$ ) mice. Firstly, we examined STAT3 activation in the livers of mice following
CBLB injection and observed a reduction of STAT3 phosphorylation in IL-22 $2^{-1-}$ mice (Fig. 4A). Furthermore, the livers of IL-22 $2^{-1-}$ mice had an attenuated transcriptional increase of the cytoprotective genes, Tnfaip3, Hmox1, and Socs3 in response to CBLB compared to wild type controls (Fig. 4B). Finally, compared to wild-type animals in which CBLB significantly decreased ALAT levels following ischemia-reperfusion, this hepatoprotective effect of CBLB was diminished in IL- $22^{-1-}$ animals (Fig. 4C). Altogether our data suggest that IL-22 is contributing to CBLB-mediated hepatoprotection.

\section{Discussion}

We show that preconditioning mice with the TLR5 agonist CBLB protected them from ischemia-reperfusion and ConA-induced liver injuries. Previous reports support the protective activity of CBLB in models of renal injury and immune-mediated hepatitis, but they focused on the immunomodulatory effect of CBLB, for example, its ability to dampen the immune response ${ }^{9,36}$. In our models, we did not observe an altered immune reactivity in mice pretreated for $2 \mathrm{~h}$ with CBLB, namely in NKT and Tcell activation markers, thus suggesting a potential increased resistance within the hepatocytes themselves to injurious stimuli. In agreement with our data, both NF- $\mathrm{kB}$ and STAT3 signaling and have been demonstrated to be activated in the liver of mice treated with $\mathrm{CBLB}^{12}$. As there is no evidence to directly link TLR5 with STAT3 activation, we investigated an indirect effect which could trigger STAT3 activation by focusing on a CBLB-induced cytokine and chemokine response. Our results show an increase in serum levels of chemoattractant (CCL2, CCL5, CXCL1, and CXCL2), pro-inflammatory (IL-1 $\alpha$, IL-6, and IL-18), and wound healing associated-cytokines (IL-5, IL22), which is in agreement with the current knowledge of a cytokine response expected following exposure to TLR5 agonists $^{37-41}$. Notably, levels of IL-22 were the highest amongst the cytokines measured. IL-22 signals through IL-22R $\alpha$ and IL-10R $\beta$, triggering STAT3 signaling and has been reported to promote liver regeneration and to decrease liver fibrosis and injury ${ }^{32-35}$. We demonstrated that IL-22 secreted after CBLB treatment was not originating from the liver, but from extrahepatic sources, namely organs upstream of the portal vein, such as colon, MLN, spleen, and intestine. This finding is strengthened by the identification of the IL-22 producing cells as $\mathrm{CD}^{-}$, $\mathrm{CD} 127^{+}$immune cells from the spleen and mucosa in response to TLR5 activation ${ }^{38}$. Based on these observations, we suppose that IL-22 produced by those visceral organs, circulates via the portal blood flow into the liver and triggers the activation of STAT3 signaling.

To better understand how CBLB could increase the resistance of hepatocytes to damage, we evaluated the effect of CBLB and IL-22 alone or in combination in 

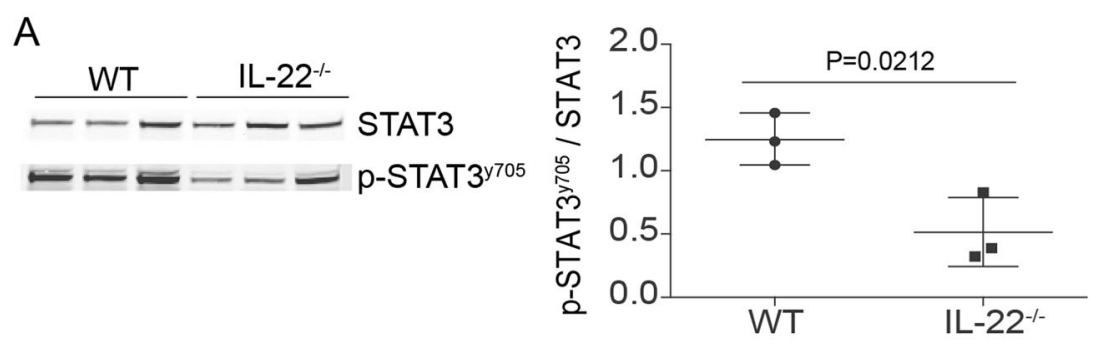

B
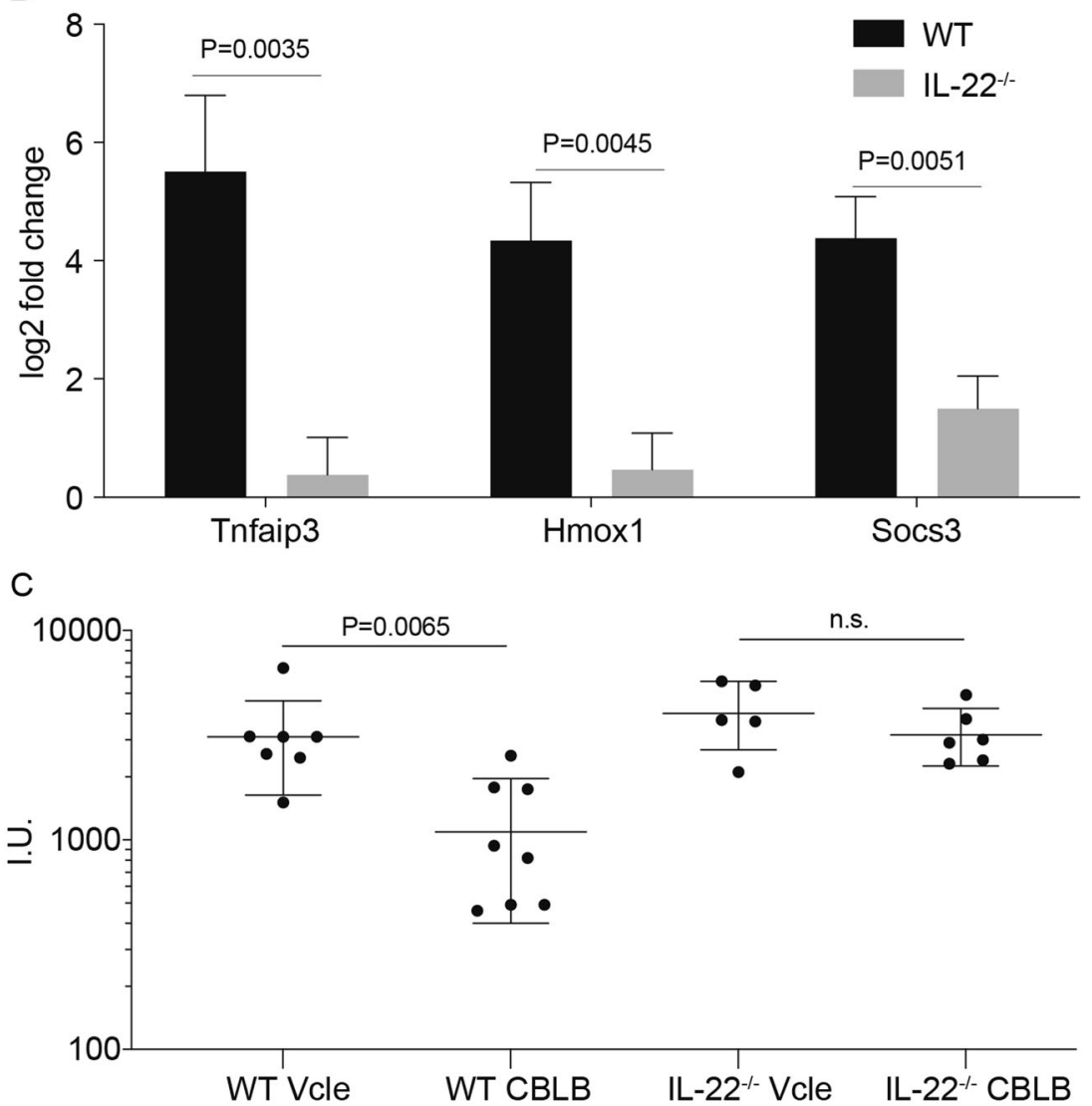

Fig. 4 IL-22 is required to mediate CBLB hepatoprotection. A Western blot analysis of proteins extracted from the livers of WT and IL-22 ${ }^{-/-}$mice for total STAT3 and STAT3 phosphorylated at tyrosine 705, $2 \mathrm{~h}$ following CBLB injection and the corresponding STAT3 phosphorylated at tyrosine 705/STAT3 ratio, $n=3$ mice per group. B qPCR of three of the cytoprotective genes (Tnfaip3, Hmox1, and Socs3) in WT and IL-22 ${ }^{-1-}$ mice $2 \mathrm{~h}$ following CBLB injection, $n=3$ mice per group. C Serum ALAT levels $6 \mathrm{~h}$ after reperfusion.

isolated mouse hepatocytes. As expected, CBLB led to the activation of the NF- $\mathrm{kB}$ pathway and resulted in a robust transcriptional response. Interestingly, the majority of the pathways affected were associated with the downregulation of genes associated with metabolism. Recent work reporting a hepatocyte-specific deletion of the TLR downstream adapter protein MyD88, also demonstrated an alteration of hepatic bile acid, glucose, and lipid metabolic functions, supporting our observations ${ }^{42}$. Although we expected to observe a strong inflammatory response, we only observed activation of the TNF signaling pathway. Through further inspection of the regulated pathways of CBLB stimulated hepatocytes, we could not show CBLB-induced signaling as responsible for the hepatoprotection we observed in vivo. IL-22R $\alpha$ is constitutively expressed by epithelial cells, including hepatocytes ${ }^{43}$. Treating primary hepatocytes with IL-22 resulted in STAT3 pathway activation; however, the transcriptomic changes were minor. The main pathway affected by IL-22 was the inflammatory response pathway, but only 18 genes were upregulated. This result was unexpected based on the proven activity of STAT3 signaling in vivo in promoting cell survival, proliferation, IRI, and ConA protection ${ }^{34,35}$. 


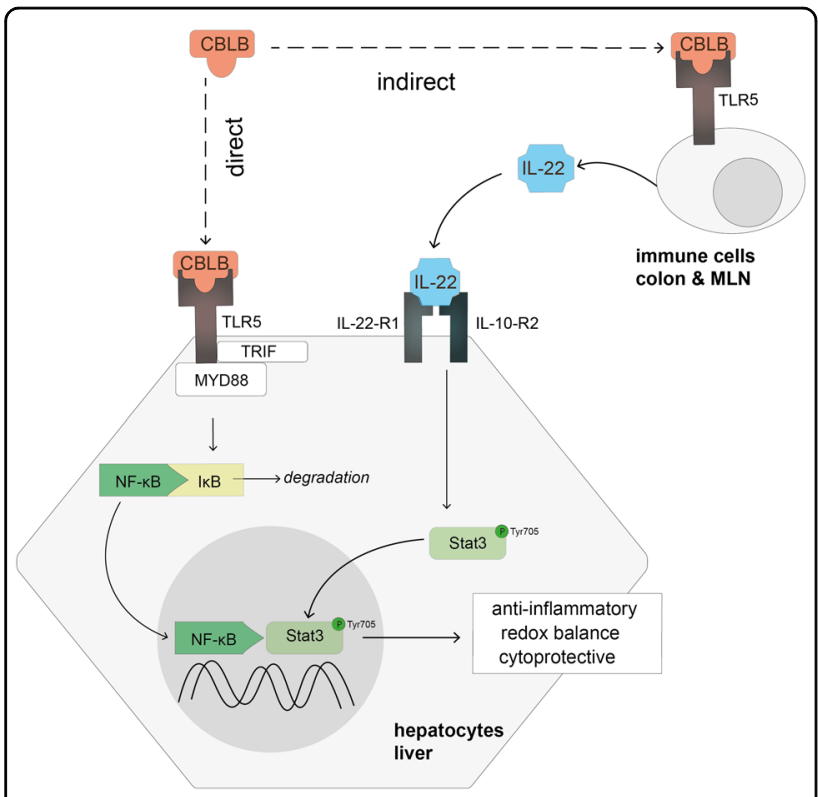

Fig. 5 Mechanism of CBLB, a TLR5 agonist, in hepatoprotection. Graphical summary of the direct and indirect action of CBLB on hepatocytes.

Often transcription factors require transcriptional cooperation in order to initiate gene transcription and interestingly, NF- $\mathrm{KB}$ and STAT3 have been largely studied for their transcriptional collaboration ${ }^{44}$. Contrary to the effect they elicit alone, the combination of CBLB + IL-22 induced a strong upregulation of genes involved in the inflammatory response pathway. The transcriptional effect of CBLB + IL-22 contains inhibitors of inflammation, apoptosis, and modulators of redox processes that are regulated following CBLB or IL-22 alone. For example, there was an upregulation of NF-kB pathway inhibitors (Nfkbia, Nfkbid, Nfkbiz, and Sbno2) that could be expected to reduce IRI damage, as NF- $\mathrm{kB}$ activation was shown to worsen the hepatic damage in IRI and ConA models ${ }^{45,46}$. In addition, the Socs protein family inhibits pathways such as insulin, IFNY TLR, and p38/JNK signaling also known to exacerbate IRI and ConA injury ${ }^{17,45-51}$. Furthermore, genes involved in redox modulation, such as Hif1a, Hmox1, Nos2, and Sod2, were shown to promote resistance in IRI models ${ }^{52}$. Finally, we identified an induction of antiapoptotic genes such as Tnfaip 3 known to promote survival in both hepatic IRI and ConAinduced hepatitis models ${ }^{53,54}$.

As the synergic treatment of CBLB and IL-22 activates a hepatoprotective transcriptional response in hepatocytes in vitro, we evaluated the difference of response of CBLB in $\mathrm{IL}-22^{-1-}$ mice. In response to CBLB, IL-22 $2^{-1-}$ mice showed a decrease of $60 \%$ in STAT3 signaling compared to WT mice. Other cytokines induced by CBLB, such as IL-6 and IL-18, are known to activate STAT signaling and might explain the remaining $40 \%$ of STAT3 phosphorylation ${ }^{55,56}$. We then confirmed in vivo the liver activation of three of the cytoprotective genes induced in hepatocytes with combined CBLB and IL-22 treatment. Tnfaip3, Hmox1, and Socs3 were strongly upregulated in the livers of WT mice, but this upregulation was absent or diminished in IL-22 $2^{-1-}$ mice. Moreover, CBLB was not able to provide the same amount of protection in the $\mathrm{IL}-22^{-/-}$ mice against ischemia-reperfusion injury. This result is concordant with the known literature on the protective activity of IL-22 $2^{34}$, TNFAIP3 ${ }^{53}$, HMOX $1^{52}$, and SOCS3 ${ }^{46-49}$ in ischemia-reperfusion injury.

Taken together, our data demonstrates that preconditioning the liver with CBLB is protective against acute hepatic injury. CBLB induces a hepatoprotective transcriptional response in hepatocytes through a direct activation of TLR5-NF- $\mathrm{kB}$ signaling in hepatocytes, as well as the induction of STAT3 signaling indirectly via the production of IL-22 from the colon and MLN (Fig. 5).

\section{Acknowledgements}

The authors would like to thank collaborators from the Laboratory of Visceral and Transplantation Surgery, Department for BioMedical Research, University of Bern for technical assistance, the Laboratory of Gastroenterology and Mucosal Immunology, Department for BioMedical Research, University of Bern for providing the MyD88/TRIF knockout mice, and the Prof. Jean-Christophe Renauld of the Ludwig Institute for Cancer Research, Catholic University of Louvain, Brussels, Belgium, for providing the IL-22 knockout mice. Finally, our sincere gratitude goes to Andrei V. Gudkov from the Department of Cell Stress Biology, Roswell Park Comprehensive Cancer Center, Buffalo, NY, USA for providing us with the CBLB502.

\section{Author details}

'Department for BioMedical Research, Inselspital, Bern University Hospital, University of Bern, 3008 Bern, Switzerland. ${ }^{2}$ Visceral Surgery and Medicine, Inselspital, Bern University Hospital, University of Bern, 3008 Bern, Switzerland. ${ }^{3}$ Department of Radiation Oncology, Inselspital, Bern University Hospital, University of Bern, 3010 Bern, Switzerland. ${ }^{4}$ Present address: Department of General, Visceral and Vascular Surgery, Bürgerspital Solothurn, 4500 Solothurn, Switzerland

\section{Author contributions}

N.M.: conceptualization, methodology, validation, formal analysis, investigation, data curation, writing the original draft, review and editing, visualization. D.S.-T. formal analysis, investigation, data curation, visualization, writing review and editing. R.F.: conceptualization, methodology, validation, formal analysis, investigation, writing review, and editing. A.K.: conceptualization, methodology, investigation, writing review and editing. M.D. and I.B.: methodology, investigation, writing review and editing. Y.Z., M.M., G.B., and D. C.: writing review and editing, resources. D.A.: writing review and editing, funding acquisition. D.S.: conceptualization, validation, writing the original draft, review and editing, visualization, resources, funding acquisition, and supervision.

\section{Funding}

The study was supported by the Swiss National Science Foundation, Grant Nr. 310030_173157 and was not involved in the study design; in the collection, analysis, and interpretation of data; in the writing of the report; and in the decision to submit the article for publication.

Data availability

RNA-seq data are available on the GEO database repository (GSE160465; link). 


\section{Ethics statement}

The study did not involve human subjects.

\section{Conflict of interest}

The authors declare no competing interests.

\section{Publisher's note}

Springer Nature remains neutral with regard to jurisdictional claims in published maps and institutional affiliations.

Supplementary information The online version contains supplementary material available at https://doi.org/10.1038/s41419-021-03654-3.

Received: 27 October 2020 Revised: 9 March 2021 Accepted: 10 March 2021

Published online: 06 April 2021

\section{References}

1. Asrani, S. K., Devarbhavi, H., Eaton, J. \& Kamath, P. S. Burden of liver diseases in the world. J. Hepatol. 70, 151-171 (2019).

2. Kiziltas, S. Toll-like receptors in pathophysiology of liver diseases. World J. Hepatol. 8, 1354-1369 (2016).

3. Huang, Q. Q. \& Pope, R. M. The role of toll-like receptors in rheumatoid arthritis. Curr. Rheumatol. Rep. 11, 357-364 (2009).

4. Lu, Y., Li, X., Liu, S., Zhang, Y. \& Zhang, D. Toll-like receptors and inflammatory bowel disease. Front. Immunol. 9, 72 (2018).

5. Arumugam, T. V. et al. Toll-like receptors in ischemia-reperfusion injury. Shock 32, 4-16 (2009).

6. Aoyama T., Paik Y. H., Seki E. Toll-like receptor signaling and liver fibrosis. Gastroenterol. Res. Pract. 192543, 1-8 (2010).

7. $\mathrm{Ha}, \mathrm{T}$. et al. Lipopolysaccharide-induced myocardial protection against ischaemia/reperfusion injury is mediated through a PI3K/Akt-dependent mechanism. Cardiovasc. Res. 78, 546-553 (2008).

8. Yoon, S. I. et al. Structural basis of TLR5-flagellin recognition and signaling. Science 335, 859-864 (2012).

9. Fukuzawa, N., Petro, M., Baldwin, W. M. 3rd, Gudkov, A. V. \& Fairchild, R. L. A TLR5 agonist inhibits acute renal ischemic failure. J. Immunol. 187, 3831-3839 (2011).

10. Hossain, M. S. et al. Flagellin, a TLR5 agonist, reduces graft-versus-host disease in allogeneic hematopoietic stem cell transplantation recipients while enhancing antiviral immunity. J. Immunol. 187, 5130-5140 (2011).

11. Burdelya, L. G. et al. An agonist of toll-like receptor 5 has radioprotective activity in mouse and primate models. Science 320, 226-230 (2008).

12. Burdelya, L. G. et al. Central role of liver in anticancer and radioprotective activities of Toll-like receptor 5 agonist. Proc. Natl Acad. Sci. USA 110, E1857-E1866 (2013).

13. Konishi, T. \& Lentsch, A. B. Hepatic ischemia/reperfusion: mechanisms of tissue injury, repair, and regeneration. Gene Expr. 17, 277-287 (2017).

14. Hoebe, $\mathrm{K}$. et al. Identification of Lps2 as a key transducer of MyD88independent TIR signalling. Nature 424, 743-748 (2003).

15. Van Belle, A. B. et al. IL-22 is required for imiquimod-induced psoriasiform skin inflammation in mice. J. Immunol. 188, 462-469 (2012).

16. Fahrner, R. et al. Tumor necrosis factor-related apoptosis-inducing ligand on NK cells protects from hepatic ischemia-reperfusion injury. Transplantation $\mathbf{9 7}$ 1102-1109 (2014)

17. Heymann, F., Hamesch, K., Weiskirchen, R. \& Tacke, F. The concanavalin A model of acute hepatitis in mice. Lab. Anim. 49, 12-20 (2015).

18. Kudira, R. et al. P2X1-regulated IL-22 secretion by innate lymphoid cells is required for efficient liver regeneration. Hepatology 63, 2004-2017 (2016).

19. Loforese, $\mathrm{G}$. et al. Impaired liver regeneration in aged mice can be rescued by silencing Hippo core kinases MST1 and MST2. EMBO Mol. Med. 9, 46-60 (2017).

20. Pfaffl, M. W. \& Hageleit, M. Validities of mRNA quantification using recombinant RNA and recombinant DNA external calibration curves in real-time RTPCR. Biotechnol. Lett. 23, 275-282 (2001)

21. Dosch M., et al. Connexin-43-dependent ATP release mediates macrophage activation during sepsis. Elife 8, e42760 (2019).
22. Rabachini, T. et al. BOK promotes chemical-induced hepatocarcinogenesis in mice. Cell Death Differ. 25, 708-720 (2018).

23. Sanchez-Taltavull D., et al. Bayesian correlation is a robust gene similarity measure for single-cell RNA-seq data. NAR Genomics and Bioinformatics 2 , 1-15 (2020)

24. Kim, D., Langmead, B. \& Salzberg, S. L. HISAT: a fast spliced aligner with low memory requirements. Nat. Methods 12, 357-360 (2015).

25. $\mathrm{Li}, \mathrm{H}$. et al. The sequence alignment/map format and SAMtools. Bioinformatics 25, 2078-2079 (2009)

26. Liao, Y., Smyth, G. K. \& Shi, W. featureCounts: an efficient general purpose program for assigning sequence reads to genomic features. Bioinformatics $\mathbf{3 0}$, 923-930 (2014)

27. Love, M. I., Huber, W. \& Anders, S. Moderated estimation of fold change and dispersion for RNA-seq data with DESeq2. Genome Biol. 15, 550 (2014).

28. Warnes G. R. et al. gplots: various $R$ programming tools for plotting data. CRANR-project https://CRAN.R-project.org/package=gplots (2019).

29. Beldi, G. et al. Natural killer T cell dysfunction in CD39-null mice protects against concanavalin A-induced hepatitis. Hepatology 48, 841-852 (2008).

30. Tallant, T. et al. Flagellin acting via TLR5 is the major activator of key signaling pathways leading to NF-kappa B and proinflammatory gene program activation in intestinal epithelial cells. BMC Microbiol. 4, 33 (2004).

31. Hillmer, E. J., Zhang, H., Li, H. S. \& Watowich, S. S. STAT3 signaling in immunity. Cytokine Growth Factor Rev. 31, 1-15 (2016).

32. Kong, X, Feng, D. Mathews, S. \& Gao, B. Hepatoprotective and anti-fibrotic functions of interleukin-22: therapeutic potential for the treatment of alcoholic liver disease. J. Gastroenterol. Hepatol. 28, 56-60 (2013).

33. Khawar M. B., Azam F., Sheikh N., Abdul Mujeeb K. How does interleukin-22 mediate liver regeneration and prevent injury and fibrosis? J. Immunol. Res. 2148129, 8 (2016).

34. Chestovich, P. J. et al. Interleukin-22: implications for liver ischemia-reperfusion injury. Transplantation 93, 485-492 (2012).

35. Radaeva, S., Sun, R., Pan, H. N., Hong, F. \& Gao, B. Interleukin 22 (IL-22) plays a protective role in T cell-mediated murine hepatitis: IL-22 is a survival factor for hepatocytes via STAT3 activation. Hepatology 39, 1332-1342 (2004).

36. Wang, L. et al. Toll-like receptor 5 signaling restrains T-cell/natural killer T-cell activation and protects against concanavalin A-induced hepatic injury. Hepatology 65, 2059-2073 (2017).

37. Descamps, D. et al. Toll-like receptor 5 (TLR5), IL-1beta secretion, and asparagine endopeptidase are critical factors for alveolar macrophage phagocytosis and bacterial killing. Proc. Natl Acad. Sci. USA 109, 1619-1624 (2012).

38. Van Maele, L. et al. TLR5 signaling stimulates the innate production of IL-17 and $1 \mathrm{~L}-22$ by CD3(neg)CD127+ immune cells in spleen and mucosa. J. Immunol. 185, 1177-1185 (2010).

39. Vijay-Kumar, M. et al. Flagellin treatment protects against chemicals, bacteria, viruses, and radiation. J. Immunol. 180, 8280-8285 (2008).

40. Chassaing, B., Ley, R. E. \& Gewirtz, A. T. Intestinal epithelial cell toll-like receptor 5 regulates the intestinal microbiota to prevent low-grade inflammation and metabolic syndrome in mice. Gastroenterology 147, 1363-1377.e1317 (2014).

41. Vijay-Kumar, M., Carvalho, F. A., Aitken, J. D., Fifadara, N. H. \& Gewirtz, A. T. TLR5 or NLRC4 is necessary and sufficient for promotion of humoral immunity by flagellin. Eur. J. Immunol. 40, 3528-3534 (2010).

42. Duparc, T. et al. Hepatocyte MyD88 affects bile acids, gut microbiota and metabolome contributing to regulate glucose and lipid metabolism. Gut $\mathbf{6 6}$ 620-632 (2017)

43. Wolk, K. et al. IL-22 increases the innate immunity of tissues. Immunity $\mathbf{2 1}$ 241-254 (2004)

44. Grivennikov, S. I. \& Karin, M. Dangerous liaisons: STAT3 and NF-kappaB collaboration and crosstalk in cancer. Cytokine Growth Factor Rev. 21, 11-19 (2010).

45. Xue, J. et al. Emodin protects against concanavalin A-induced hepatitis in mice through inhibiting activation of the p38 MAPK-NF-kappaB signaling pathway. Cell Physiol. Biochem. 35, 1557-1570 (2015).

46. Sherif, I. O., Al-Shaalan, N. H. Vildagliptin attenuates hepatic ischemia/reperfusion injury via the TLR4/NF-kappaB signaling pathway. Oxid. Med. Cell Longev. 2018, 3509091 (2018).

47. Li, X. L. et al. Insulin in UW solution exacerbates hepatic ischemia / reperfusion injury by energy depletion through the IRS-2 / SREBP-1c pathway. Liver Transpl. 10, 1173-1182 (2004).

48. Kobayashi, M., Takeyoshi, I., Yoshinari, D., Matsumoto, K. \& Morishita, Y. P38 mitogen-activated protein kinase inhibition attenuates ischemia-reperfusion injury of the rat liver. Surgery 131, 344-349 (2002). 
49. Uehara, T. et al. JNK mediates hepatic ischemia reperfusion injury. J. Hepatol. 42, 850-859 (2005).

50. Evankovich J., Billiar T., Tsung A. Toll-like receptors in hepatic ischemia/reperfusion and transplantation. Gastroenterol. Res. Pract. 2010537263 (2010).

51. Ojiro, K. et al. MyD88-dependent pathway accelerates the liver damage of Concanavalin A-induced hepatitis. Biochem. Biophys. Res. Commun. 399, 744-749 (2010).

52. Elias-Miro, M., Jimenez-Castro, M. B., Rodes, J. \& Peralta, C. Current knowledge on oxidative stress in hepatic ischemia/reperfusion. Free Radic. Res. 47, 555-568 (2013).
53. Ramsey, H. E. et al. A20 protects mice from lethal liver ischemia/reperfusion injury by increasing peroxisome proliferator-activated receptor-alpha expression. Liver Transpl. 15, 1613-1621 (2009).

54. Zilberman-Rudenko, J. et al. Recruitment of A20 by the C-terminal domain of NEMO suppresses NF-kappaB activation and autoinflammatory disease. Proc. Natl Acad. Sci. USA 113, 1612-1617 (2016).

55. Alboni, S. et al. Interleukin 18 activates MAPKs and STAT3 but not NF-kappaB in hippocampal HT-22 cells. Brain Behav. Immun. 40, 85-94 (2014).

56. Taub, R. Hepatoprotection via the IL-6/Stat3 pathway. J. Clin. Invest. 112, 978-980 (2003) 